\title{
La préface en philosophie : une approche discursive
}

The Preface in Philosophy: A Discursive Approach

\section{Frédéric Cossutta}

\section{(2) OpenEdition}

Journals

\section{Édition électronique}

URL : http://journals.openedition.org/aad/2990

DOI : 10.4000/aad.2990

ISSN : 1565-8961

Éditeur

Université de Tel-Aviv

\section{Référence électronique}

Frédéric Cossutta, "La préface en philosophie : une approche discursive », Argumentation et Analyse du Discours [En ligne], 22 | 2019, mis en ligne le 15 avril 2019, consulté le 23 septembre 2019. URL : http:// journals.openedition.org/aad/2990 ; DOI : 10.4000/aad.2990

Ce document a été généré automatiquement le 23 septembre 2019.

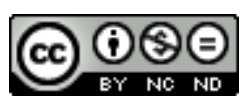

Argumentation \& analyse du discours est mis à disposition selon les termes de la licence Creative Commons Attribution - Pas d'Utilisation Commerciale - Pas de Modification 4.0 International. 


\title{
La préface en philosophie : une approche discursive
}

The Preface in Philosophy: A Discursive Approach

\author{
Frédéric Cossutta
}

« La préface, acte premier par lequel commence à s'établir la monarchie de l'auteur » ${ }^{1}$

1 Je me propose d'aborder la question des préfaces en philosophie ${ }^{2}$, moins en termes de seuil, de « liminarité » ou de frontière que dans la perspective d'une activité discursive spécifique, celle d'un "agir préfacier" ${ }^{3}$. On aurait pu en effet se référer à la classification proposée par Gérard Genette ${ }^{4}$ qui a le mérite de rendre compte, par une série de critères croisés, de la complexité de l'activité préfacière, mais sa typologie demeure descriptive. A propos d'un type de discours non littéraire, et sans avoir la prétention d'offrir une meilleure classification, je voudrais reprendre la réflexion sur cette question, en proposant une investigation qui étalonne les fonctions préfacières sur des critères différents, en l'occurrence sur des propriétés discursives d'ordre pragmatique.

2 Les préfaces opèrent en effet un certain nombre de transactions et de réglages préliminaires à la mise en forme d'un livre en articulant simultanément le texte qu'elles inaugurent à ses dimensions compositionnelles internes (cotexte) et à certaines instances discursives externes (contexte). Elles jouent un rôle crucial en accomplissant un premier nouage entre certaines dimensions fondamentales qui concourent à l'élaboration du discours philosophique - lequel, pour ne fonder sa validité que sur la recherche explicite de procédures d'accès au vrai, n'en doit pas moins satisfaire un certain nombre d'impératifs communicationnels et, dès lors, concilier des exigences qui pourraient sembler contradictoires ou au moins sources de tensions. Le discours théorique doit répondre simultanément à ces deux types d'exigences, tantôt par la recherche d'une proportion équilibrée, tantôt en privilégiant l'une sans faire totalement l'impasse sur la seconde, au prix de réglages et de choix qui supposent parfois une stratégie délibérée. Pour répondre à ces tensions, la préface, à côté d'autres segments textuels ou paratextuels, élabore une scène discursive associant étroitement 
les schèmes spéculatifs d'une doctrine philosophique ou d'une théorie à vocation scientifique (avec ses structures et procès d'analyse, ses méthodes d'investigation), à ses schèmes expressifs (modes d'exposition, choix génériques, dimensions figuratives). J'ai proposé un certain nombre de catégories sur lesquelles je m'appuierai pour analyser la façon dont les préfaces mettent en place des protocoles de transaction et d'ajustement entre instauration discursive et institution discursive d'un corps théorique ${ }^{5}$.

3 L'hypothèse que je désire ici mettre à l'épreuve est que les préfaces ont pour fonction discursive de contribuer à surmonter certains paradoxes pragmatiques qui se posent aux discours constituants (Maingueneau et Cossutta 1995, Cossutta 2015a).

Pour concilier ces contraintes paradoxales, les préfaces et textes liminaires effectuent trois types de transactions ${ }^{6}$ nécessaires à l'élaboration d'un livre à vocation théorique ${ }^{7}$. L'examen de ces transactions constituera le fil directeur de cette contribution :

5 - Transactions sur le rapport entre le texte et son contexte (seuil externe du livre). Elles concernent les relations entre le texte et le hors texte, la construction d'une identité auctoriale, corrélative d'un positionnement dans le champ. Cette fonction auctoriale distribue des places, incorpore des voix, figure un ethos, en corrélation directe avec la définition des destinataires (lecteur, postérité, auditoire universel).

6 - Transactions sur le rapport entre le texte et son co-texte (seuil interne du livre). Elles définissent des paramètres ouvrant un espace-temps textuel qui offre un support au déploiement d'un parcours théorique dont il faut instituer le commencement et contrôler le développement jusqu'à son terme. Identité auctoriale et identité doctrinale s'élaborent corrélativement avec le déploiement d'une structure interne du livre que la préface organise, à côté des subdivisions, titrages, récapitulations, résumés, guides de lecture, index et table des matières.

7 - Transactions sur le rapport entre le texte et l'œuvre (opus/corpus, doctrine/œuvre). La préface, sur le seuil d'une œuvre singulière (opus) tisse des liens avec l'ensemble de l'œuvre d'un auteur (corpus). Elle procède à une mise en relation avec les œuvres antérieures, explicite des continuités, des ruptures, des bilans, des réponses aux objections etc.

\section{La préface articule le texte au hors texte ou contexte}

\subsection{La préface élabore une identité auctoriale et un personnage de philosophe}

8 Une première série de transactions permet d'articuler le texte et son contexte d'inscription éditoriale, institutionnelle aussi bien qu'intellectuelle. La préface est un lieu d'interface entre extériorité et intériorité. Elle définit un rapport entre le texte et son contexte en élaborant la frontière entre l'interne et l'externe, entre lesquels elle doit construire une relation en boucle. Pour ce faire, elle installe une scène particulière, à la fois en surplomb et en immanence par rapport au corps de l'ouvrage. On peut se demander en effet qui parle dans une préface. Est-ce la même instance énonciative que celle qui prend en charge les analyses tout au long de l'ouvrage? Non, dans la mesure où la préface suppose un décalage inversé par rapport au texte qu'elle semble anticiper. 
Loin de le préfigurer, elle en est une post-figuration puisqu'elle ne peut s'écrire que dans l'après-coup de l'œuvre dont elle se présente pourtant comme le préambule. Elle suppose une voix en surplomb, celle par laquelle une personne se construit comme auteur de son texte et producteur de son livre.

La préface, grâce au décalage opéré par l'instance énonciative, confère à une doctrine une forme d'existence «objectivable» en la nommant, en l'inscrivant dans la matérialité du livre qui en rend possible la saisie, la mention, le commentaire. Par autoréférence ("Dans cette préface», "Dans ce livre..., », "J’ai écrit ce livre...»), en le nommant par son titre (nombre de préfaces commencent par une glose du titre) ou en lui assignant une signature emblématique qui pose son identité théorique: «Je suis heureux de pouvoir parler de la phénoménologie transcendantale [...] », écrit Husserl à la première ligne des Méditations cartésiennes (1929/1966: 1). Elle donne, au seuil du livre, la représentation d'une extériorité associée à sa matérialité éditoriale et économique tout comme à son identification juridique. Ce rapport extériorisant est reconstruit de l'intérieur du livre par les références éditoriales, l'évocation des circonstances de rédaction et de publication, les renvois à des circonstances chronologiques, institutionnelles avérées. Il devient possible de manipuler le livre comme une entité matérielle et idéelle ayant une consistance propre et une autonomie tout en le situant par rapport à autre chose que lui-même : on peut s'y référer, le citer. Cette observation générale n'est certes pas spécifique à la préface philosophique, mais celle-ci a pour particularité de devoir reconfigurer, à l'occasion d'une nouvelle publication, l'architecture d'un univers doctrinal préconstruit.

Corrélativement à la position d'un énonciateur à la source du texte, la préface permet de poser le statut surplombant d'un auteur du livre (comme entité juridique et comme personnage). Elle permet dès lors d'embrayer simultanément une position d'auteur associée à son ethos, et la construction d'une image de ses destinataires. Ce passage d'une version non retenue de la préface à la seconde édition du Gai savoir, où Nietzsche sculpte sa propre statue de penseur incompris, en est un très bon exemple :

Mais le but de cette préface en doit être un autre que celui de rappeler à mes lecteurs ce que sont les vertus d'un lecteur: bonne volonté, réflexion, attention, compréhension, perspicacité ; ce serait pure simulation, si je me bornais à cela. Je sais trop bien pourquoi ce livre sera mésentendu(sic) ou, plus exactement, pourquoi son presque arbitraire plaisir à la clarté, à l'immédiat, à la légèreté, à l'insouciance ne se communique point, agit plutôt en tant que problème, et, en tant que problème, inquiète... (1886 [1982] : 331, développe la note 1 de la page 21).

11 Il faut bien cependant surmonter, fût-ce ironiquement, la discordance entre les attentes par avance déçues à l'égard de la réception du livre et le fait qu'on l'écrive cependant, en affectant d'en attendre la compréhension de la seule postérité. Ce qui peut se faire de deux façons : par le bais de la métaphore : " et si vous ne comprenez pas, si vous comprenez mal le chantre, qu'importe! C'est la "malédiction du chantre". En revanche, vous entendrez d'autant plus nettement sa musique et sa mélodie, et son pipeau vous fera d'autant mieux-danser. Le voulez-vous ?...» (ibid.: 293, fin du §383); ou par le biais de considérations d'ordre méta-préfacier: "Ce livre aurait sans doute besoin de plus d'une préface; en fin de compte, subsistera toujours le doute que quelqu'un, pour n'avoir rien vécu d'analogue, puisse jamais être familiarisé par des préfaces avec l'expérience préalable à ce livre. » (ibid. : 22, \$1). 


\subsection{La préface opère un positionnement dans le champ}

Comment ne s'autoriser que de soi-même tout en tenant compte des autorités instituées ou des garants extérieurs de la véracité? Les préfaces déploient des trésors de subtilité stratégique pour effectuer ce positionnement dans le champ et par rapport à l'archive déposée par une tradition : trop insister sur la dette, c'est manquer d'originalité et s'inféoder à une école, trop insister sur la rupture, c'est courir le risque de l'isolement et de l'incompréhension ${ }^{8}$. Comment modifier par la publication la configuration de ce champ et y faire reconnaître, et si possible dominer, la position théorique que l'on propose? En le re-présentant dans une scène préfacière qui en reconfigure l'image. Celle-ci propose un tableau de la situation institutionnelle ou disciplinaire dans laquelle s'inscrit l'auteur, en reformulant les débats et les conflits entre les acteurs en cause. Elle dessine une certaine image de soi du penseur comme extérieur à l'institution philosophique ou au contraire comme ayant vocation à s'inscrire pleinement comme acteur dominant du champ, à travers un jeu complexe d'affiliations et de démarquages.

La difficulté n'est pas la même selon qu'on se trouve dans une situation où la régulation de l'ordre discursif est assurée par les instances politiques et religieuses qui se prévalent d'un droit de regard sur la « vérité » en exerçant un magistère institutionnel ou que l'on s'inscrit dans un jeu concurrentiel dont les rapports de force se stabilisent à travers des dispositifs universitaires, éditoriaux, médiatiques ou commerciaux. Ainsi la première édition latine de 1641 des Méditations métaphysiques de Descartes, comporte un paratexte complexe. Elle s'ouvre sur une page titre dans laquelle le titre ne dit pas seulement le genre de l'ouvrage mais aussi sa teneur (Méditations de Philosophie Première où se trouve démontrée l'existence de Dieu et l'immortalité de l'âme), suivie d'une approbation quelque peu usurpée puisque Descartes ne l'avait pas reçue : "Avec Privilège et Approbation Des Docteurs ». Vient en second lieu une épître dédicatoire «A Messieurs les Doyens et Docteurs de la Sacrée Faculté de Théologie de Paris », puis une "Préface de l'auteur au lecteur». Le paratexte de l'édition en français de 1661 modifie ce dispositif qui obéissait à une stratégie d'investissement du champ pour laquelle Descartes était prêt à faire toutes les concessions nécessaires, et en particulier à varier les cadres génériques à travers lesquels exposer sa métaphysique9 .

En revanche, dans un contexte tout à fait différent, un auteur contemporain comme $\mathrm{P}$. Bourdieu dans Le sens pratique (1980) procède à ce positionnement grâce à une série de démarcations théoriques par rapport aux courants et auteurs qui balisent le paysage de la philosophie et des sciences humaines contemporaines. Il s'agit là d'une reconstruction ou d'une reconstitution dans laquelle on distribue les rôles et les positions en reconstruisant le champ extérieur par rapport auquel le livre se détache, et cela en fonction même des catégories sociologiques et des dispositifs spéculatifs mis en place par le texte qu'on va lire. Bourdieu les met en acte en quelque sorte performativement dans la préface et dans les autres séquences paratextuelles. Il anticipe aussi bien les conditions de production que les conditions de réception de ses ouvrages en fonction de sa théorie du capital intellectuel et du statut du chercheur en relation avec une modélisation sociologique des pratiques universitaires et éditoriales ${ }^{10}$.

15 Cette intériorisation du champ, dont l'effet attendu est sa transformation, est anticipée sur la réception du livre dans les «préfaces auctoriales originales » pour la faciliter et 
écarter les incompréhensions, alors que les préface "tardives" de seconde ou troisième éditions intègrent les critiques et les mésinterprétations qu'elles s'efforcent de rectifier ou d'interpréter à leur tour (Genette 1987 : 177, 182). Elles mesurent les effets de la publication comme le montre le cas des deux préfaces au traité de Schopenhauer, Le Monde comme volonté et comme représentation ${ }^{11}$. Par contraste, un " iconoclaste » comme Kierkegaard assume un décalage par rapport aux réceptions de ses livres en usant de l'ironie, par exemple lorsqu'il fait l'apologie de la mésinterprétation ou lorsque, parfaitement cohérent avec la préface aux Riens philosophiques, il fait état, dans son adresse finale au lecteur en appendice du Postscriptum aux miettes philosophiques publié un an plus tard, de l'absence totale d'écho éveillé par la publication du petit opuscule.

\subsection{La préface met en place les mécanismes de la destination ou de l'adresse}

La préface prend en charge d'autres difficultés qui portent sur la relation au destinataire : comment concilier la visée d'un auditoire ciblé, particulier, et celle d'un public indifférencié, d'un auditoire universel ? Dans le premier cas il faut jouer sur une familiarité contextuelle (les allusions par exemple aux personnages et circonstances historiques qu'un lecteur lointain ne comprendra plus sans un apparat critique). Dans le second, il faut faire en sorte qu'elle puisse valoir pour un auditoire élargi supposé hors contexte (ce qui ne veut pas dire hors de tout contexte mais dont le contexte ne peut être anticipé). Les préfaces diversifient leurs cibles et formatent l'adresse selon qu'elles privilégient un mode d'exposition didactique et pédagogique ou au contraire qu'elles prennent le parti de heurter le lecteur ou au moins de ne pas lui faciliter la tâche, comme c'est le cas avec Kierkegaard et Nietzsche. Jules Vuillemin, par exemple, dans Nécessité ou contingence. L'aporie de Diodore et les systèmes philosophiques présente de façon sèche, sobre et rigoureuse sa démarche en résumant de façon lapidaire son objet et son plan détaillé : « Les paradoxes de Zénon portent sur le continu et le mouvement. L'aporie de Diodore porte sur les idées de nécessité et de contingence. Ce livre a pour objet d'étudier ces idées en partant de cette aporie. Il est divisé en quatre parties" (1984: 7). Ni le contexte ni le lecteur ne sont pris en compte sinon de façon allusive (ibid. : 10).

Un même auteur, lorsque l'adresse est explicite, dans deux préfaces chronologiquement distinctes pour le même livre ${ }^{12}$, peut délimiter une cible différente et moduler son adresse en fonction du type de destinataire convoqué. Dans sa première préface de 1819 à Le Monde comme volonté et comme représentation, Schopenhauer s'adresse personnellement au lecteur du livre en le guidant péremptoirement dans sa lecture : «Si l'on veut lire ce livre de la manière qui en rend l'intelligence aussi aisée que possible, on devra suivre les indications ci-après » (1819 [2006]:1). Mais il modifie son adresse dans la seconde, beaucoup plus tardive (1844), sur un ton où perce à la fois l'amertume, le ressentiment mais aussi l'espoir de voir le temps lui donner enfin un vrai lectorat: " Ce n'est pas à mes contemporains, ce n'est pas à mes compatriotes, c'est à l'humanité que j'offre mon œuvre cette fois achevée, dans l'espérance qu'elle en pourra tirer quelque fruit [...]. Il est vrai que le public, tout ce temps durant, n'y a pas pris intérêt» (1844 [2006]: 9). L'auteur, entre humour et sarcasme, conseille en désespoir de cause au lecteur rétif d'utiliser le volume comme décoration dans sa 
bibliothèque ou comme cadeau sur la table d'ouvrage d'une dame ou, pourquoi pas, d'« en faire un compte rendu critique » (ibid.).

\section{La préface structure la forme du livre et amorce le mouvement d'auto-constitution du discours théorique (rapport entre la préface et son cotexte)}

\subsection{La préface assure la constitution d'un espace-temps interne unifié}

La préface est placée sur l'entame constitutive de l'espace-temps du livre mais ne peut en pratique être écrite, ou du moins parachevée, qu'une fois celui-ci terminé. Certaines jouent le jeu de l'anticipation purement programmatique, d'autres se placent résolument dans une posture rétrospective, selon le rapport qu'elles instaurent avec la temporalité interne du livre. Les segments liminaires doivent donc à la fois assurer la fonction d'ouverture et de clôture du discours en délimitant par avance son espacetemps interne, en définissant son formatage et éventuellement sa composition.

Il s'agit de produire le mouvement de la pensée dans sa substance propre, la matérialité discursive où elle s'inscrit ${ }^{13}$, qui définit un espace-temps textuel normé par des règles de circulation interne liée aux exigences auxquelles elle doit s'adapter pour être communicable. Mais comment construire une totalité textuelle dont tous les moments soient idéalement co-présents, puisque l'organisation des dépendances et hiérarchies structurelles entre concepts, thèses, dispositifs spéculatifs, parties et sous-parties d'ensembles doctrinaux, construite idéalement en surplomb, ne peut s'énoncer que séquentiellement dans une linéarité orientée liée au dispositif matériel du support écrit ${ }^{14}$ ? Les préfaces et textes liminaires, ainsi que de nombreux éléments du paratexte (repères chiffrés, découpages en paragraphes et en séquences titrées et numérotées, index et tables des matières, renvois internes), permettent de contrebalancer l'irréversibilité matérielle et temporelle du processus de constitution du sens dans l'ordre linéaire de la lecture ${ }^{15}$. Les grilles de numérotation, le découpage, les tables de référence (index, tables des matières) et les multiples renvois internes permettent des parcours obligés liés à un mode d'emploi (parfois explicite) auxquels s'ajoutent ceux que le lecteur peut effectuer librement en feuilletant le volume selon son gré ${ }^{16}$. La préface, en particulier, donne les échelles et les boussoles ainsi que le tempo d'un trajet de lecture et de pensée, en même temps qu'elle dispose par anticipation l'esquisse d'une cartographie conceptuelle.

La préface propose une entame, un incipit, mais tout autant anticipe la forme de l'œuvre, avec le risque de paradoxes logiques: un sous-ensemble qui intègre une représentation de l'ensemble dont il fait partie, une image du tout à l'intérieur du tout qu'elle reflète, comme une mise en abîme non figurative qui mimerait ce qu'elle veut anticiper tout en en explicitant les caractéristiques: en un double sens la préface « réfléchit » le livre.

On peut se demander à ce propos s'il existe une constante isomorphie tant sur le plan des modes d'expression que sur celui des opérations de pensée entre les préfaces et le corps du texte. On peut le vérifier assez souvent, notamment par le biais d'études comparatives. Ainsi, une modification doctrinale associée à une modification de la 
forme, du genre textuel ou du style se reflète aussi bien dans la préface que dans le texte. On le voit très nettement en comparant l'écriture respective des préfaces et du livre dans le cas du Tractatus logico- philosophicus de Wittgenstein, dont la préface est extrêmement assertive et lapidaire: "la vérité des pensées communiquées ici me paraît intangible et définitive " (1914 et 1945 [1961] : 28) et celle des Investigations philosophiques qui évoque des pensées dispersées, «résidus d'investigations philosophiques [...], je ne les ai rédigées qu'en tant que remarques, en de brefs paragraphes $[. .]. »(111)^{17}$.

\subsection{La préface tente de résoudre la question du commencement de l'acte spéculatif}

Une série de difficultés porte sur la nécessité d'ancrer l'autonomie, l'identité, la stabilité d'une entité théorique par son inscription dans une unité de discours matériellement organisée. L'entre-deux entre le commencement et la fin du livre n'est pas seulement l'espace-temps matériel du support, mais aussi la temporalité propre au déploiement de schèmes spéculatifs ou théoriques dans lesquelles s'effectuent les opérations de pensée.

Un des paradoxes les plus intéressants est celui qu'on pourrait nommer le paradoxe du commencement : comment concilier le caractère relativement contingent, d'un point de vue historique, des circonstances individuelles ou personnelles de rédaction et d'édition d'un ouvrage et la prétention à l'objectivité, à l'universalité d'un propos caractérisé par la nécessité et l'auto-conscience ? La préface doit lier commencement $\mathrm{du}$ livre et commencement de la spéculation. Elle opère une transaction entre la contingence de l'inscription éditoriale, biographique ou historique du texte et la visée d'universalité ou de scientificité à laquelle prétendent nombre de doctrines qui doivent dès lors expliciter les modalités de leur constitution et les critères de leur véridicité. On observe une variation corrélative entre type de préface et nature de la doctrine. Descartes, Kant, Husserl posent la question du commencement en termes d'absence de présupposition, d'effacement ou de neutralisation de la subjectivité du penseur. «Il devient nécessaire", écrit Husserl au seuil de ses Méditations cartésiennes, "de reconstruire l'édifice qui pourrait correspondre à l'idée de la philosophie, conçue comme unité universelle des sciences s'élevant sur un fondement de caractère absolu " (1929 [2006] : 2). Il lui faut dégager un sol originaire : "Par conséquent le véritable problème du début est celui-ci : quelles sont les vérités premières en soi qui devront et pourront soutenir tout l'édifice de la science universelle ?» (Ibid.: 8) Les philosophies de l'histoire, comme le montre la préface à la Phénoménologie de l'esprit de Hegel ${ }^{18}$ ou certains textes de Feuerbach et de Marx doivent penser le commencement comme une forme d'émergence qui échappe à sa propre historicité tout en n'étant pensable qu'à partir d'un point de vue situé dans l'histoire. De leur côté, les philosophies qui refusent toute idée de fondement, comme celles de Nietzsche ou de Kierkegaard, font face à un problème inverse : la contingence du commencement cette fois est assumée, sur le modèle d'un surgissement créateur impromptu, sur celui d'une révélation inspirée ou d'une transgression ironique et comique. Le risque, cette fois, porte moins sur le commencement que sur la fin. La question cruciale devient : Comment finir? Par où finir ? Comment éviter la répétition, le bégaiement ou le radotage (penser à la solution proposée par Kierkegaard dans La répétition ${ }^{19}$ ou à Ecce Homo et à la « fin » de Nietzsche) 
? Le discours peut devenir in-terminable, comme le montre le Post-scriptum aux Miettes philosophiques de Kierkegaard, énorme complément du minuscule opuscule intitulé en français Miettes ou Riens philosophiques.

\section{Le rôle de la préface dans la constitution d'une « œuvre » philosophique}

La préface accompagne le mouvement d'auto-constitution d'une philosophie ou d'une théorie aux visées généralisantes en les inscrivant dans la singularité d'une œuvre. Elle ouvre un univers d'idéalités, un régime conceptuel et idiomatique structurés selon des schèmes spéculatifs qui l'identifient comme un corps théorique nouveau. En tant qu' opus chaque livre est inscrit dans un horizon plus large, celui du corpus d'un auteur qui est éventuellement aussi une œuvre ou un chef d'œuvre pouvant figurer dans le panthéon d'un patrimoine immatériel de l'humanité. La préface contribue à inscrire le texte dans le continuum ou les inflexions et trajectoires d'une identité doctrinale qui se joue et se transforme parfois en se remettant en jeu dans les différents moments de sa manifestation éditoriale. Comment concilier ces deux plans où se conjuguent nécessité et aléa ? La préface y contribue fortement car elle procède à une articulation organique entre opus et corpus, entre corpus et œuvre. Chaque livre (opus) peut prendre la forme d'une œuvre philosophique à part entière, mais comme dans un univers romanesque, les différents livres eux-mêmes peuvent s'intégrer en une œuvre conçue comme totalité, fût-elle non systématique voire fragmentaire. Ainsi on retrouve au gré de la lecture les mêmes «personnages conceptuels $»^{20}$. Les formes de production du discours théorique obéissent simultanément à ces impératifs logiques de cohérence spéculative et à des contraintes aléatoires liées aux circonstances éditoriales.

\subsection{Elaboration d'une identité doctrinale ou théorique et diversité de ses inscriptions éditoriales (liaison opus/corpus)}

Comment concilier le fait qu'en droit une doctrine est une, ou au moins sous-tendue par une nécessité interne qui relie ses évolutions, reformatages et transformations, voire ses propres reniements et qu'en même temps la multiplicité de ses manifestations discursives et de ses moments d'inscription éditoriale soit dispersée et souvent soumise à la contingence? Cela pose la question du rapport entre les différentes unités de signification, dotées d'une relative autonomie et qui pourtant s'agencent plus ou moins bien, de façon plus ou moins réglée pour offrir une configuration théorique d'ensemble qui prend souvent, en philosophie, la forme du système. Les préfaces jouent ici encore un rôle essentiel, dans la mesure où elles relient les différents éléments de l'œuvre à la fois d'un point de vue chronologique et d'un point de vue structurel. Elles assignent leur place respective aux ouvrages publiés ou à paraître. Une activité théorique se développe à travers les occurrences plus ou moins circonstancielles de ses diverses manifestations chronologiquement répertoriées : formes orales, conférences, entretiens, cours, correspondances, publications, interventions circonstancielles dans la presse ou sous forme d'interviews ${ }^{21}$. La préface a pour fonction de concilier la contingence et l'hétérogénéité des formes éditoriales de production du discours avec l'architecture théorique qui se projette dans le plan des idéalités, que cet univers de discours soit totalement conscient et définitivement fixé, ou qu'il se cherche à tâtons à 
travers le coup de dé de chaque nouvel opus. Nous avons observé dans les exemples proposés plus haut les déplacements effectués dans les préfaces d'un même livre au cours de ses rééditions chronologiques (préfaces tardives). De même, les préfaces de différents livres opèrent souvent une mise en relation synoptique des œuvres antérieures de l'auteur. Elles peuvent aussi anticiper les œuvres à venir. Il arrive assez souvent que la forme systématique donnée dans les préfaces de façon anticipée ou programmatique à un ensemble spéculatif subisse des transformations, des déformations, parfois des distorsions lors de sa mise en œuvre. Certains textes liminaires sont alors chargés de réaménager le dispositif d'ensemble ou de résoudre les risques de contradictions latentes. C'est ce que fait la première Introduction à La Critique de la faculté de juger de Kant qui tente d'harmoniser le système des trois Critiques inscrites dans trois ouvrages qui ne sont pas écrits simultanément.

Deux formes principales d'homogénéisations rétrospectives permettent à l'auteur de situer l'un par rapport à l'autre ses écrits et sa théorie. La première peut être autobiographique, lorsqu'il associe les étapes de son parcours philosophique à des évènements intimes ou publics ${ }^{22}$. Nous avons vu Wittgenstein dans sa seconde préface poser des jalons temporels datés pour situer son évolution philosophique depuis la publication du Tractatus.

L'autre biais est architectonique, lorsque les introductions et préfaces catégorisent dans les termes même d'une doctrine le rapport qui existe entre les différents ouvrages en effaçant ce qui est dû aux circonstances de rédaction et d'édition au profit d'une organisation raisonnée, systématique ou éclatée. Kant, par exemple, lorsqu'il écrit l'avant-propos des Fondements de la métaphysique des mœurs a déjà à l'esprit l'architecture globale de son système, mais doit justifier les tâtonnements qui l'ont conduit à publier les Fondements de la métaphysique des mœurs : «Or, dans l'intention où je suis de publier un jour une métaphysique des mœurs, je la fais précéder de ce livre qui en pose les fondements. Sans doute il n'y a à la rigueur pour pouvoir la fonder, qu'une Critique d'une raison pure pratique comme pour fonder la Métaphysique il faut la Critique de la raison pure spéculative que j'ai déjà publiée» (Kant 1785 [1993]: 55). C'est précisément la fonction de la section III de l'Introduction de la Critique du jugement que de penser la notion de système : «Il est très important de déterminer exactement quelles sont les parties de la philosophie, et, à cette fin, de ne pas inscrire parmi les membres de sa division comme système ce qui n'en constitue qu'une conséquence ou une application données, sans requérir pour cela des principes particuliers [...]» (1790 [1995] : 89).

\subsection{Intertextualité et autonomie préfacières}

Les préfaces peuvent aussi entrer en résonance et se reprendre ou se répondre entre elles, comme si elles formaient un intertexte préfacier transversal à un corpus. Une préface tardive est en relation intertextuelle explicite avec les précédentes, comme nous l'avons vu à propos de Schopenhauer: "Déjà, dans la préface de la première édition, j'ai déclaré que ma philosophie procède de celle de Kant...» (1819 [2006]:15). Parfois la relation est implicite, par exemple lorsqu'une seconde préface destitue la précédente sans la nommer ou détruit l'idée même de préface, comme le fait celle de la seconde édition de l'Histoire de la folie de Michel Foucault. Il s'agit ici d'une méta-préface anti-préfacière ${ }^{23}$. La relation interpréfacière peut même avoir lieu entre les œuvres de 
philosophes différents. Ainsi la courte préface de Kierkegaard aux Miettes philosophiques joue-t-elle le rôle d'une anti-préface puisqu'elle prend le contre-pied de la préface de Hegel à la Phénoménologie de l'esprit, tant du point de vue du style que du propos.

Parfois même elles peuvent exister de façon autonome comme des textes réunis pour eux-mêmes. A titre exceptionnel certaines préfaces ou introductions ont une autonomie de sens suffisante pour être lues pour elles-mêmes, comme le montre l'existence de livres de commentaire associés au seul texte de la préface à la Phénoménologie de l'esprit. Il n'existe pratiquement pas, à ma connaissance, de recueils de préfaces de philosophie ou de sciences humaines comme il en existe en littérature où leur rôle de manifeste explique leur possible autonomisation éditoriale ${ }^{24}$.

On rencontre cependant des livres réunissant des préfaces fictives, comme c'est le cas de Borges dans Le livre des préfaces (1975 [1980]). Genette fait allusion à un petit opuscule de Nietzsche offrant à Cosima Wagner Cinq préfaces à cinq livres qui n'ont pas été écrits (1987 : 239). Kierkegaard a écrit un livre de préfaces imaginaires à des livres non écrits, façon de théoriser sur le mode d'une communication indirecte et de façon ironique les questions liées aux difficultés de réception de son œuvre philosophique (1844 [1973]).

\subsection{Le rôle des préfaces dans le devenir-œuvre ou chef-d'œuvre d'une philosophie (de la doctrine à l'œuvre)}

31 La préface, en tissant ces liens interdiscursifs internes à un univers de pensée, contribue dès lors au « devenir-œuvre » d'une théorie ou d'une philosophie puisqu'elle réassigne la place respective des éléments de discours par rapport à la forme doctrinale d'ensemble, que cette forme soit éclatée ou compacte, en lui donnant une configuration globale emblématique et reconnaissable entre toutes, parfois condensée dans une formule ${ }^{25}$.

Un exposé doctrinal ou d'histoire de la philosophie de Descartes, Spinoza ou Leibniz pourrait à la limite être rédigé selon un mode d'exposition systématique et canonique à structure analogue (qui expliciterait leur conception de Dieu, du monde physique, du rapport âme- corps, etc.). Mais si l'on compare la façon dont les philosophies de Descartes et de Leibniz font œuvre (c'est peut-être déjà beaucoup dire pour Leibniz), on ne peut manquer d'être frappé par la différence: on observe d'un côté la relative cohérence formelle de l'œuvre cartésienne (la stabilité de son formatage au plan des schèmes spéculatifs) élaborée dans un éventail de cadres génériques très diversifié mais homogène en dépit de la contingence des circonstances de son énonciation, et de l'autre la dispersion éditoriale, le foisonnement générique, la diversité des circonstances et des types de textes par lesquelles Leibniz, le philosophe européen en constant déplacement, en perpétuel conciliabule scientifique, théologique ou diplomatique, multiplie les entrées et les angles d'approche dans un système protéiforme et hétérogène dont on serait bien en peine de donner la structure ou une caractérisation globale en tant qu'œuvre ${ }^{26}$.

Mais en quel sens peut-on parler d'une «œuvre» en philosophie? Pourquoi donner une consistance à cette catégorie, alors que le terme de théorie, de corps doctrinal, rapporté à un corpus constitué et répertorié, semble suffire ? En effet, la catégorie d'« œuvre " semble nous déporter, au-delà des conditions de bonne formation d'un discours, au-delà des visées aléthiques et éthiques d'un corps d'énoncés de doctrine, 
vers une dimension esthétique qui vaut sans doute pour les œuvres artistiques et littéraires mais paraît difficilement applicable ici sans aménagement. On pourrait imaginer conférer à un livre de philosophie le statut d'une œuvre voire d'un chefd'œuvre en étalonnant cette évaluation sur des critères formels et surtout discursifs (liés à certaines modalités du rapport existant entre forme du contenu et forme de l'expression). Je ne peux développer ici cette question ${ }^{27}$ qui supposerait de distinguer plusieurs sens du terme "œuvre». Mais on voit très bien comment les préfaces travaillent à ce devenir-œuvre d'une pensée, en nouant la singularité d'une identité spéculative et celle d'un style d'écriture dans un ensemble composé (organique, sans que la règle de composition soit nécessairement celle du système) et pas seulement composite.

\section{Conclusion}

\section{Méta-préfaces, hyper-préfaces, anti-préfaces} œuvre, les préfaces accomplissent fréquemment un retour réflexif sur le statut de l'œuvre (à la fois texte singulier et corpus d'un auteur), sur son devenir une fois qu'elle sera ou a été publiée, ce qui conduit à observer une dimension métalangagière dans nombre de préfaces qui thématisent leur propre statut de préface. J'appelle "métapréface » une préface qui se thématise elle-même en tant que préface et/ou qui explicite les propriétés des préfaces en général. Rien d'étrange à cela: les discours philosophiques, en tant que discours constituants, sont auto-constituants, et cela est valable quel que soit le mode d'exposition et de régime de pensée choisi, systématique, anti- ou a-systématique (Cossutta 2015a: 68). Ils sont par conséquent conduits à thématiser leurs modes de légitimation dans les termes même des catégories qu'ils élaborent, avec les distorsions que cela peut produire ${ }^{30}$. Dès lors il est normal que l'on trouve dans les préfaces les effets de cette boucle réflexive, qu'elle soit assumée explicitement chez les uns ou "forclose " chez les autres. Cette dimension réflexive dans les textes liminaires se traduit par la présence d'une dimension «métapréfacière » qui peut se manifester ponctuellement, comme l'a montré la préface à la seconde édition du Gai savoir, ou parfois même structurer une préface tout entière, comme c'est le cas pour la Phénoménologie de Hegel ou les Miettes philosophiques de Kierkegaard.

Ce qui est en jeu dans le mécanisme méta-préfacier est donc précisément le rapport qu'entretient ce sous-ensemble avec le livre et l'œuvre comme forme et comme tout. 
Tantôt par excès, lorsque des hyper-préfaces posent le livre comme « absolu », totalité effective et définitivement achevée (Ethique de Spinoza, Tractatus de Wittgenstein, Phénoménologie de l'esprit de Hegel, Sens pratique de Bourdieu). Tantôt par défaut, lorsqu'elles dénoncent dans des anti-préfaces le livre comme une totalité impensable, ou comme une imposture qui doit laisser place à des traces ou à des formes plus légères ou plus composites. Pensons à certaines préfaces de Nietzsche, Kierkegaard, Foucault ou Derrida qui précèdent des textes hybrides.

La dimension méta-préfacière peut ainsi servir deux fins opposées. Elle peut traduire le plus haut degré d'intégration des schèmes expressifs aux schèmes spéculatifs, les premiers étant de part en part asservis aux seconds. La préface est alors chargée d'opérer une boucle réflexive sur la boucle réflexive, le livre réabsorbant son mode de constitution et les conditions extérieures de sa construction dans l'effort qu'il fait pour procéder à une explicitation intégrale qui se veut "sans reste", ce qui le promeut comme « Le Livre » ultime. A l'opposé, elle peut s'efforcer de traduire le plus haut degré d'intégration, (il faudrait plutôt dire de désintégration) des schèmes spéculatifs dans les schèmes expressifs ${ }^{31}$. Dans un tel cas, le livre se déconstruit dans le mouvement même où il s'écrit, comme sous le coup d'un « effet Pénélope » qui le détisse à mesure qu'il se trame, comme si la teneur philosophique ou théorique qu'il proposait n'était pas compatible avec le fait d'écrire un « livre », du moins selon les canons traditionnels de la philosophie et qu'était exigé un déplacement vers des formes nouvelles d'hybridation et de littérarité qui deviennent une médiation nécessaire pour le développement d'une pensée « inouïe ».

\section{BIBLIOGRAPHIE}

Barton, Ben F. \& Marthalee S. Barton. 1993. «Preface/Metapreface », Journal of Business and Technical Communication 7, 3-11

Borges, Jorge Luis. 1975 [1980]. Livre de préfaces suivi de Essai d'autobiographie (Paris : Gallimard)

Bourdieu, Pierre. 1980. Le Sens pratique (Paris : Minuit)

Bourdieu, Pierre. 1986. "L'illusion biographique », Actes de la Recherche en Sciences Sociales 62, 1, 69-72

Bourdieu, Pierre. 2001. Science de la science et réflexivité (Paris : Editions Raisons d'Agir)

Bourdieu, Pierre. 2004. Esquisse pour une auto-analyse (Paris : Editions Raisons d'Agir)

Cossutta, Frédéric. 1995. « Pour une analyse du discours philosophique », Langages 119,

«L'analyse du discours philosophique », 12-39

Cossutta, Frédéric. 1996. « Argumentation, ordre des raison et mode d'exposition dans l'œuvre cartésienne », Cossutta, F. (éd.). Descartes et l'argumentation philosophique (Paris : PUF), 111-185

Cossutta, Frédéric. 2001. «Les formes en philosophie », Cahiers philosophiques 89, "Styles et genres en philosophie », 65-81

Argumentation et Analyse du Discours, 22 | 2019 
Cossutta, Frédéric. 2005. « Discours philosophique, discours littéraire : le même et l'autre ?» Rue Descartes 50, «L'écriture des philosophes », 6-20

Cossutta, Frédéric. 2015 a. «Les "discours constituants" vingt ans après », Angermuller Johannes \& Gilles Philippe. Analyse du discours et dispositifs d'énonciation. Autour des travaux de Dominique Maingueneau (Limoges : Lambert-Lucas), 61-78

Cossutta, Frédéric. 2015 b. « Éperons le style de Nietzsche, un texte indéchiffrable?», Maingueneau, Dominique \& Mathilde Vallespir (éds). Lire Derrida ? (Limoges : Lambert-Lucas), 14-41

Cossutta, Frédéric \& Francine Cicurel (éds). 2012. Les formules philosophiques (Limoges : LambertLucas)

Cossutta, Frédéric, Pascale Delormas \& Dominique Maingueneau (éds). 2012. La vie à l'œuvre. Le biographique dans le discours philosophique (Limoges : Lambert-Lucas)

Deleuze, Gilles \& Félix Guattari. 1991. Qu'est-ce que la philosophie? (Paris : Minuit)

Foucault, Michel. 1972. Histoire de la folie à l'âge classique (Paris : Gallimard)

Genette, Gérard. 1987. Seuils (Paris : Seuil)

Husserl, Edmond. 1929 [1966]. Méditations cartésiennes. Introduction à la phénoménologie (Paris : Vrin)

Kant, Emmanuel. 1785 [1993]. Fondements de la métaphysique des mœurs (Paris : Le livre de Poche)

Kant, Emmanuel. 1790 [1995]. Critique de la faculté de juger (Paris : GF Flammarion)

Kierkegaard, Søren. 1844 [1973]. Préfaces, Euvres complètes, vol. 7 (Paris : Editions de l'Orante), 259-326

Maingueneau, Dominique. 2015. La philosophie comme institution discursive (Limoges : LambertLucas)

Maingueneau, Dominique \& Frédéric Cossutta. 1995. "L'analyse des discours constituants » Langages 117, « Les analyses du Discours en France », 112-125

Nietzsche, Friedrich 1882 et 1887 [1982]. Le gai savoir (Paris : Gallimard)

Nietzsche 1886 [1999]. Essai d'autocritique et autres préfaces, présenté, traduit et commenté par Marc de Launay (Paris : Seuil)

Schaeffer, Jean-Marie. 1987. « Note sur la préface philosophique », Poétique 69, 35-44

Schopenhauer, Arthur. 1844 et 1819 [2006]. Le monde comme volonté et comme représentation (Paris : PUF)

Vuillemin, Jules. 1984. Nécessité ou contingence. L'aporie de Diodore et les systèmes philosophiques (Paris : Minuit)

Wittgenstein, Ludwig. 1914 et 1945 [1961]. Tractatus logico-philosophicus, suivi de Investigations philosophiques (Paris : Gallimard)

\section{NOTES}

1. Foucault $1972: 10$

2. Cet article s'appuie sur une recherche qui prend également en considération le statut des préfaces en sciences humaines, mais je me concentre ici sur le discours philosophique et je me limite à l'étude des préfaces auctoriales. 
3. J'utilise ici le terme de "préface" dans un sens générique pour désigner les séquences textuelles liminaires d'un livre et n'entrerai pas dans les distinctions entre avertissement, avantpropos, préface, introduction car elles dépendent, en situation, du nom parfois interchangeable qui leur est donné lorsqu'un seul texte liminaire est proposé. Par contre la distinction préface/ introduction devient pertinente lorsque deux ou plusieurs séquences d'ouverture se juxtaposent en tête de l'ouvrage, au point qu'il faudrait parler de " paratexte liminaire » pour rendre compte de la diversité des incipit. On rencontre des constructions assez complexes, comme par exemple dans le dispositif préfacier du livre de Bourdieu, Le sens pratique (1980), qui comporte une «Préface générale » éditée en italique et un «Avant-propos» pour chacun des deux livres qui composent l'ouvrage, sans oublier la quatrième de couverture qui obéit à un type de régime paratextuel comparable à celui de la préface.

4. Le livre de Genette, Seuils, donne une classification des préfaces devenue classique. Il utilise une série de critères croisés : moment de publication (" originale », "ultérieure », "tardive » [1987 : 185 et 189]), nature du destinateur (distinction entre "autographe » ou "auctoriale ", " actoriale ", " allographe »), distinction entre " authentique », " apocryphe » et " fictive ». Sur les préfaces en philosophie, voir aussi Schaeffer (1987).

5. Pour un éclairage sur ces catégories voir Cossutta $(1995,2001)$.

6. J'entends par transaction la façon dont l'auteur opère de façon consciente ou non un calcul et des ajustements concernant les proportions accordées à l'une ou l'autre des contraintes discursives que les préfaces ont pour fonction de concilier.

7. Je les distingue pour clarification, dans les faits elles s'intriquent selon des modalités et des proportions variables.

8. Que l'on songe à la façon dont Derrida détourne dans nombre de ses livres la reconnaissance de dette, par exemple au seuil de Eperons, les styles de Nietzsche (cf. Cossutta 2015 b : 18-19).

9. Pour une étude du rapport entre ces stratégies éditoriales et les modifications des dispositifs génériques qu'elles requièrent chez Descartes, voir Cossutta 1996.

10. Cette boucle réflexive est caractéristique des discours constituants, qu'ils aient une vocation fondatrice, comme c'est le cas pour le statut scientifique que Bourdieu veut donner à la sociologie (Bourdieu 1986, 2001), ou qu'ils en récusent la possibilité.

11. Voir l'exemple développé ci-dessous.

12. Dans les éditions posthumes les différentes préfaces se cumulent en tête du même ouvrage.

13. Le changement de support, notamment le passage à un support électronique, modifie la donne, puisque le déroulement sur écran réintroduit certaines caractéristiques de la lecture antérieure aux volumes imprimés ou manuscrits. Mais la multiplication des liens hypertextuels et des renvois intertextuels ou des tables et index "actifs » brouillent les frontières de ce qui constituait jusqu'à présent les délimitations et la singularité d'une œuvre.

14. Un philosophe comme Derrida, reprenant indirectement le travail d'Apollinaire ou de Mallarmé sur la disposition typographique du poème, joue, par exemple dans « Thympan » ou Glas, sur les dispositions graphiques de la page imprimée. Il déjoue, ce faisant, les règles de composition d'une unité de signification et d'exposition philosophique classique, en procédant à des collages de commentaires, de citations, de segments textuels typographiquement différenciés qui ouvrent à un mode de lecture complètement différent et à une appréhension disséminée de l'écriture.

15. Une des nouveautés majeures liée à l'utilisation de supports matériels télématiques est l'affaiblissement des frontières entre unités textuelles, l'effacement des identités auctoriales, la difficulté d'assignation des sources, la prolifération textuelle permettant d'envisager des productions foisonnant de proche en proche, multi-centrées et poly-auctoriales.

16. J'ai essayé de montrer comment le dispositif déductif de l'Ethique de Spinoza s'accompagne de boucles de rétroaction démonstrative et de niveaux et chemins de lecture multiples (Cossutta 1995).

Argumentation et Analyse du Discours, 22 | 2019 
17. Une analyse attentive de ces enchaînements (ou ceux des Carnets ou autres ouvrages liés à ces cours), en apparence aléatoires, montre qu'ils sont cependant déterminés par certaines règles de composition, malgré leur rédaction au jour le jour, d'où le nom d'«Album » que donne cette préface à leur récollection.

18. Un dédoublement des textes liminaires peut aussi correspondre à des fonctions différentes. Ainsi La phénoménologie de l'esprit, pour le dire rapidement, se voit anticipée simultanément par une Préface et une Introduction. La première prélude au Système (à partir d'un point de vue rétrospectif, celui du «Savoir Absolu») dont l'ouvrage fait partie à côté d'autres œuvres, alors que l'Introduction prépare La phénoménologie de l'esprit en tant qu'ouvrage particulier.

19. Les éditions récentes traduisent le titre, de façon plus fidèle et explicite, par La reprise.

20. J'emprunte cette expression à Qu'est-ce que la philosophie? de Deleuze et Guattari, en manière de simplification (1991).

21. Pensons au rôle comparable qu'assurent: - les curriculum vitae en ligne qui déclinent chronologiquement les étapes d'une carrière, - les bibliographies qui proposent un inventaire des publications ou interventions, - les listes de publications classées chronologiquement ou en fonction de critères universitaires, - les autobiographies intellectuelles, les entretiens, les conférences, les correspondances peuvent aussi contribuer à donner un point de vue synoptique ou rétrospectif sur une œuvre. Ces reconfigurations narratives d'une œuvre ou d'une carrière constituent certes elles aussi un élément du corpus mais dans un décalage logique ou chronologique, alors que les préfaces et postfaces sont des unités de signification liées en immanence au livre qu'elles préludent même si elles le surplombent. Elles exercent un travail plus organique de nouage entre les œuvres en articulant in vivo chaque nouvel opus au précédent et à l'ensemble déjà constitué, ou éventuellement aux prochains, parfois en fonction d'une structure idéale dont certaines places encore vides définissent un horizon de tâches à venir.

22. Sur le rôle des dimensions biographiques dans l'élaboration d'un discours philosophique, voir Cossutta, Delormas et Maingueneau (éds). 2012.

23. Voir ci-dessous, la définition des méta-préfaces.

24. Une exception : un recueil de certaines préfaces de Nietzche de 1886 sont rassemblées en un volume par Marc de Launay, parce qu'elles sont reliées par une profonde continuité de sens (1886 [1999])

25. Concernant l'expression formulaire en philosophie voir Cossutta, Cicurel (éds). 2014.

26. Certes M. Serres s'y est essayé avec un certain bonheur en parlant du Système de Leibniz et ses modèles mathématiques ", ou G. Deleuze en utilisant l'image du pli.

27. Pour une esquisse, voir Cossutta $2005: 19$.

28. Elles devraient être mises à l'épreuve sans trop d'aménagement à propos des Sciences humaines et sociales.

29. On rencontre une grande diversité d'agencements liminaires et de compositions de ces séquences ; les analyser orienterait à présent l'investigation vers une dimension typologique que nous ne pouvons pas développer ici, cet article faisant partie d'un travail inédit qui aborde l'ensemble des questions posées par l'étude des préfaces philosophiques et de sciences humaines. 30. Un exemple emblématique de ce bouclage réflexif peut être observé chez Hegel ou dans la façon dont la sociologie de Bourdieu pense les conditions de sa propre légitimité en thématisant dans ses propres catégories les conditions de son émergence et de son développement (Bourdieu 1986, 2001, 2004).

31. On pourrait nommer hyper-préface et anti-préface ces deux formes de méta-préfaces extrêmes (pour les SHS, voir Barton et Barton 1993). 


\section{RÉSUMÉS}

En mobilisant certaines catégories élaborées dans le cadre de ma conception de l'Analyse du Discours philosophique, je m'attache à montrer, sur un objet apparemment secondaire, les préfaces et texte liminaires en philosophie et sciences humaines, la fécondité de cette méthode. Je déplace l'analyse descriptive et typologique des seuils, élaborée par Genette, au profit de l'étude pragmatique d'un "agir préfacier » déterminant pour l'auto-constitution des discours théoriques. Les préfaces opèrent certaines transactions discursives permettant de surmonter le risque de tensions entre les contraintes liées aux visées de vérité et celles qui relèvent de visées communicationnelles. Elles relient en boucle le texte à son contexte et à son cotexte, négocient le mode d'inscription simultané de l'auteur et du lecteur ainsi que les conditions de la lecture, configurent l'organisation interne du livre, relient opus et corpus et contribuent à donner à une doctrine la forme de l'œuvre, voire du chef-d'œuvre.

By using some categories developed within the framework of my own conception of philosophical discourse analysis, I aim to demonstrate the productivity of such an approach on a corpus that may seem peripheral: forewords and opening texts in philosophy and Human Sciences. Instead of drawing on Gerard Genette's description of text "thresholds", I develop a pragmatic approach of "forewording action", which plays a key role in the auto-constitution of theoretical discourses. Forewords achieve discourse transactions that allow the authors to reduce the risk of tensions between truth constraints and communication constraints. They link in a reflective way texts and their linguistic and extralinguistic contexts, negotiate the way authors and readers are anchored in texts, specify the conditions of reading, configure the inner organization of the book, tightly connect opus and corpus, and help to shape a doctrine as a work - and even as a masterpiece.

\section{INDEX}

Keywords : corpus, foreword, Human and Social Sciences, masterpiece, opus, philosophical discourse analysis, self-constituting discourses, work

Mots-clés : Analyse du Discours philosophique, chef-d'œuvre, corpus, discours constituant, discours de Sciences humaines, œuvre, opus, préface

\section{AUTEUR}

\section{FRÉDÉRIC COSSUTTA}

CEDITEC, Paris-Est Val de Marne 